\title{
Integration of the Open Government Data platform into existing Content Management Systems in Kenya
}

\author{
Dorothy Gatwiri Bundi \\ Department of Computer Science \\ Kenya Methodist University, Meru, Meru County, Kenya \\ Amos Chege Kirongo \\ Department of Computer Science and Information Technology \\ Meru University of Science and Technology, Meru, Meru County, Kenya
}

\begin{abstract}
Open Government Data (OGD) avails statistical, development, expenditure and demographic data, in digital format for the general public, ICT developers, researchers and policy makers. This evaluation is based on analysis of qualitative and quantitative metadata and data sets in Open Data portals. The OGD platform integration to Content Management Systems, targeted internal and external users of Open Data portals in Kenya. The implementation of the OGD platform is facing a number of challenges resulting from the government ministries and county governments reluctance in releasing secretive information. To realize the benefits of OGD which includes transparency, public service improvement, innovation and economic value, efficiency, self-empowerment, impact measurement of policies, participatory governance, and data mining new knowledge from combined data sources and patterns from large datasets; the government needs to integrate OGDs in CMS. This paper analyses theoretical and empirical open data sets and proposes an integration framework of OGD to CMS. This paper analyses theoretical and empirical open data sets and proposes recommendations for future integration framework of Open Government Data strategies and Platforms as part of Content Management Systems. The paper outlines the benefits of OGD integration initiatives to CMS in Kenya. The innovation compliments government processes by introducing citizen-centered portals to enable government to design and distribute open data allowing interactions and seamless responses from the public. OGD is currently in use and can be scaled down to County Governments Open Data portals.
\end{abstract}

Keywords - Content Management System, Data Sets, Integration, Open Government Data, Metadata Portals

\section{INTRODUCTION}

The Kenya Open Government Data Initiative was launched and relaunched in 2011 to enable increase access to government datasets (Mutuku \& Mahihu, 2014). Governments and public institutions across the world collect, generate, store and disseminate broad range of data in order to perform their day to day tasks(Janssen, Charalabidis, \& Zuiderwijk, 2012; Yunliang, Xiongtao, Qing, Jing, \& Ning, 2010). This has led to the need of sharing public sector data and information, which is aimed at achieving public sector accountability, reduction of operational costs, efficiency in service delivery, quality of the services offered and innovations (Bertot, Jaeger, \& Grimes, 2010).

Open government data relating to public services e.g. the waiting time of an office visit for the renewal of driver's license, is currently enabled in the Kenyan governments using the e-citizen content management systems (Nixon Kanali, 2015).

The main aims of the OGDs initiatives is to make the government agencies more transparent participative and collaborative, encouraging pubic involvements in data collection, cleaning, analysis, presentation and application for economic growth (Parycek, Höchtl, \& Ginner, 2014).

As per the Second Medium Term Plan 2013-2017, the government of Kenya has flagship project initiatives aimed at the development of local digital content including multimedia local content, e-government, e-learning, e- 
health care, e-commerce, e-marketing, and information and data gathering, retrieval, storage and communication (Government of Kenya, 2013).

The public has benefited from the implementation of OGDs through increased data transparency, accountability, increased awareness of government activities and programs resulting to public collaborations, innovations and creation of value-added services (Janssen \& Estevez, 2013). In addition, this has improved decision-making processes among the government officers and her citizen.

According to (Ubaldi, 2013) the availability and openness of the government databases accessed via web and mobile applications is expected to improve the quality of lives and the economy due to increased access to the government datasets when making choices.

Whereas there has been benefits in the implementation of OGDs, there still exists challenges which include; lack of harmonized data management system, inadequate information resource centers, limited penetration of telecommunication infrastructure in rural areas coupled with the digital divide between the rural and urban areas which limits public awareness of the advantages and opportunities of OGD (Darrel, 2015).The rest of the paper is organized as follows. The state of OGD in Kenya in section II. The method is presented in section III. Discussion is done in section IV. Concluding remarks are given in section V.

\section{THE STATE OF OGD IN KENYA}

\section{A. Definition}

Open Government Data (OGD) is defined as "datasets, selected by a governmental institution, which are structured and provided in a machine-readable format for machine-readability." ("Sebastian Meumanier, Open Data Quality Assesment and Evaluation of (Meta-) Data Quality in the Open Data Landscape," n.d.). These datasets provide non-confidential and non-privacy restricted data, published in online catalogs accessible through search engines and other data mining tools. Open Data in Kenya, can be found in the opendata.go.ke portal of the Kenyan Government.

Content Management System (CMS) is a web application system that allows publishing, editing, and modifying of web-based workflow procedures and content as well as site maintenance from a central page(Burdon, 2009; Esperança \& Pereira, 2016).

Metadata. (Eden, 2009) defines "metadata as data about data, and can is expressed on three ways: embedded metadata that is contained in the markup of the resource itself, associated metadata maintained in files tightly coupled to the resources they describe, and third-party metadata maintained in a separate repository by an organization that may or may not have direct control over or access to the content of the resource."

\section{B. OGD Development in Kenya}

Opendata.go.ke portal was launched in 2011, becoming the first Open Government Data portal in Africa. The portal is accessible through mobile applications and third party web. Every Kenyan Citizen has the right to access state owned information as per the Artilce 35 of the Bill of Rights(Government of Kenya, 2013). During the launch of the opendata.go.ke portal, there were over 200 datasets, later increasing to over 430 datasets in two years, with over 176,000 page views, and over 5500 datasets downloaded and embedded to several blogs and websites(Government of Kenya, 2013).

\section{METHOD}

\section{A. The Study Design}

This paper is geared to evaluating the success and the barriers of OGD implementation and the need of integrating them to CMS. Several methods were involved including analysis of secondary literature of internationally published OGD publications. Different perspectives have been drawn stating the benefits, barriers, facts, myths of open data in aiding governments initiates and innovations.

Document analysis has also been used to provide insight for government departments and bodies responsible for execution of the OGD policies. Operative documents which can be downloaded to statistically to show datasets, APIs and their origins. 
Additionally, empirical Quantitate and Qualitative Analysis of secondary datasets from Open Data Portals based on internal and external user access to OGDs and CMS has been used.

The areas evaluated in this paper include; the status of OGDs implementation, the benefits and barriers of OGDs integration, and the proposed strategies that can be used to integrate OGDs to CMS.

\section{B. Population and sample}

The study is based on some government departments which include, e-government, e-learning, e-health and ebusiness. The data analyzed is from the open data portals datasets showing the importance of integrating OGDs with the existing CMS. Various variables have been used to evaluate the government initiatives of allowing her citizens to freely access and distribute open data

\section{IV.DISCUSSION}

\section{A. Status of OGD implementation in kenya}

\section{Legal framework and Policies}

Existing open data analyzed from the World Bank show that the population in Kenya currently stands at 46.1 Million with an annual population growth of $2.4 \%$. Further $42 \%$ of the population are of the age $0-14,55 \%$ are of the age 15-64 whereas only $3 \%$ of the population is of age 65 and above. The analysis shown that $30 \%$ of the population is aware of the legal and policy frameworks that exist to support the OGD usage whereas the bigger part of the population is not aware of the existence of the OGD supporting policies.

Numerous policy challenges are an obstacle to the success of OGD initiatives. The lack of clear policies pose a threat to data transparency, copyright issues and security restriction of the public in using the government data. Lack of guidelines, standards and procedures of how to create, validate, share and deal with the open data compromises the quality of the data available on OGD platforms.

Over time the availability and accessibility of relevant OGD weakens in terms of format and content leading to low interest by the public in use of OGD, hence the need to improve the legal frameworks that will encourage the public to access and share the open data.

For example, the Kenya Institute of Curriculum Development has digitized content for classes 3 to 8 in science and mathematics for primary education in Kenya. Two frameworks exists namely the ICT for Education and the National ICT Innovation and Integration Centre which are involved in spearheading the pedagogical use of ICT and testing of technical solutions for use in curriculum development. There also exists an ICT integration model, which encompasses four pillars for effective implementation of ICT initiatives aimed at developing teacher capacity, development of relevant digital content, and rolling out of ICT infrastructure and robust policy and strategy. The aim these initiatives by the government is to increase access to quality digital educational materials, whereas the public is not fully informed of this state.

It is important to have legal and policy frameworks and strategies that support OGD in conjunction with the CMS and ensure they are known and adopted by the citizens.

\section{Technical issues}

Over $90 \%$ of government department have their services and process supported by ICT which results to a pool of knowledge that can be made open and freely accessed via the CMS. The analysis revealed less than $30 \%$ of the public is aware of the existence of OGD in Kenya hence government departments and agencies can be facilitated to carry out training to the public enhancing the awareness of existence of open government data. The government ICT and geographical information processing affiliated departments collects, stores and distributes the OGD GIS data. The CMS can establish links to geo servers which store OGD metadata with the actual data sets.

The open data is availed, accessed and reused in open and machine-readable formats. This open data can be accessed and downloaded using any web browsers and search engines.

The analysis revealed more than $60 \%$ of the departments that use modern and integrated ICT systems show readiness to share and publicize data as OGD. This also allows cross departmental exchange of e-government information supported by CMS. This enables departments to be autonomous in their decision-making process. 


\section{Economic and financial}

A cost and benefit analysis show that the use of CMS to enable access of OGD results to reduced operational costs, increased efficiency, value added services, quality of life and economic growth. $70 \%$ of the analyzed financial reports shown that if the government adopts the use of integrated OGD to CMS it would result to accountability of government agencies to its citizen which will increase the investments and trust.

World bank statistics analyzed from open data indicate that $19.3 \%$ of gross capital formation of the Kenyan financial status is aid depended from developed countries, while $12.4 \%$ of goods were imported in the year 2014 . This information is available in the World Bank open data portal while missing in the Kenyan OGD portal.

\section{Organizational Implementation}

The decision to implement OGDs as part of the existing CMS was proved to be more advantageous than standalone OGD platforms with more than $50 \%$ of the ICT department dataset showing that the availability of the digitized content from the existing CMS is available online, hence no extra training is required to enable the users access the open data due to previous knowledge of the use of CMS.

More than $90 \%$ of the government e-services are supported by the CMS making it easy for government to provide, control and monitor access to government data.

\section{Communication and Interaction}

The analysis indicated that $65 \%$ of the people who accessed OGD services proposed that there is need to develop an ecosystem where the public can be enlightened and educated on the importance of engaging and evaluating the government initiatives through OGD. The public will be enabled to engage with the wider community by sharing the feedback on relevance of the OGD datasets. These engagements will result to high public satisfaction and interaction through embedded platforms supported by the government CMS.

The government CMS promotes access to OGD even for the minorities and disabled. Over $50 \%$ of Public and private partnerships have actively encouraged innovation and enabled public participation in using OGD and improve policy making process.

The OGD platforms have interfaces that when integrated with CMS will make it easier to access help functionalities and allow the users to give feedback.

The review of the available datasets also shown that OGD can be supported by the social media platforms which will accelerate the access of the government data.

\section{B. Benefits and Barriers of OGD Implementation}

The implementation of OGD has resulted to various political, economic and social benefits (Buzzi, Ferrucci, Gennai, \& Petrucci, 2016; Janssen et al., 2012; Janssen \& Estevez, 2013; Origlia, Cersosimo, Bianchi, \& Fortunato, 2016; Weseni, Watson, \& Anteneh, 2015) and the tangible benefits of the freely released government data certainly overshadows its challenges. Bertot et al states that scrutiny of the metadata show key benefit in value added services with easier access and increased number of the users that participate in the OGD (Bertot et al., 2010), increased transparency of public processes and administration leading to informed citizens who can engage in the monitoring, control and decision making processes, automated government administration processes which results to reduced administration costs and efforts, accountability by Government officers resulting to better quality of life and economy, and high productivity and economic growth enabling new innovations and creativity. OGD initiatives have increased collaborations and sharing of datasets among the public and private organizations increasing the user satisfaction and reducing the error reporting by users.

On the other hand the implementation of OGD has faced some challenges that include: Loss of income due to freely released data. Lack of knowledge about the existence of OGD by the public hence less usage. Other challenges featuring in the analysis are Policy, technical, legal, organizational, cultural, economic and financial challenges (Janssen et al., 2012).

The policies sometimes limit data transparency and copyrights of who owns the government data. This can restrict the public's right to use and distribute the government data. The public also may confuse the right to access information and the right to freedom of expression 


\section{OGD Integration Strategies}

The citizen-centered portals is one of the strategy that can be used to enable government to design and distribute open data. A citizen-centered portal will allow interaction, seamless responses and accountability among the OGD stakeholders. The OGD frameworks can also be structured in a transparent way to enable government promote transparent services meeting the needs of its citizen. Flattening the organizational structures using the online portals that transform the processes from vertical forms where departments acts independently to horizontal forms where departments share common information. (Parycek et al., 2014)

Service Oriented Architecture (SOA) is another strategy that can be used to create OGD platforms that can be used to share government applications (Nasr, Gross, \& van Deursen, 2010). The flexibility and power of SOA enables government to create and install new services across different departments and platforms. SOA enables integration of other systems to the OGD portals, since the use of SOA components reduces complexity of the system and increases reusability (Lee \& Kwak, 2011; Nasr et al., 2010).

Call for collaboration (CFC) is a strategy that the government can use to invite the individuals and private companies to submit proposals that can be used to develop and deploy OGD portals. Competition can be used to create awareness of the government e-services and the open datasets (Chan, 2013).

\section{Proposed OGD-CMS Integration Framework}

An Analytical Framework for national OGD integration to CMSs supported by online portals and Government Initiatives has been proposed showing the success and barriers of the implementation.

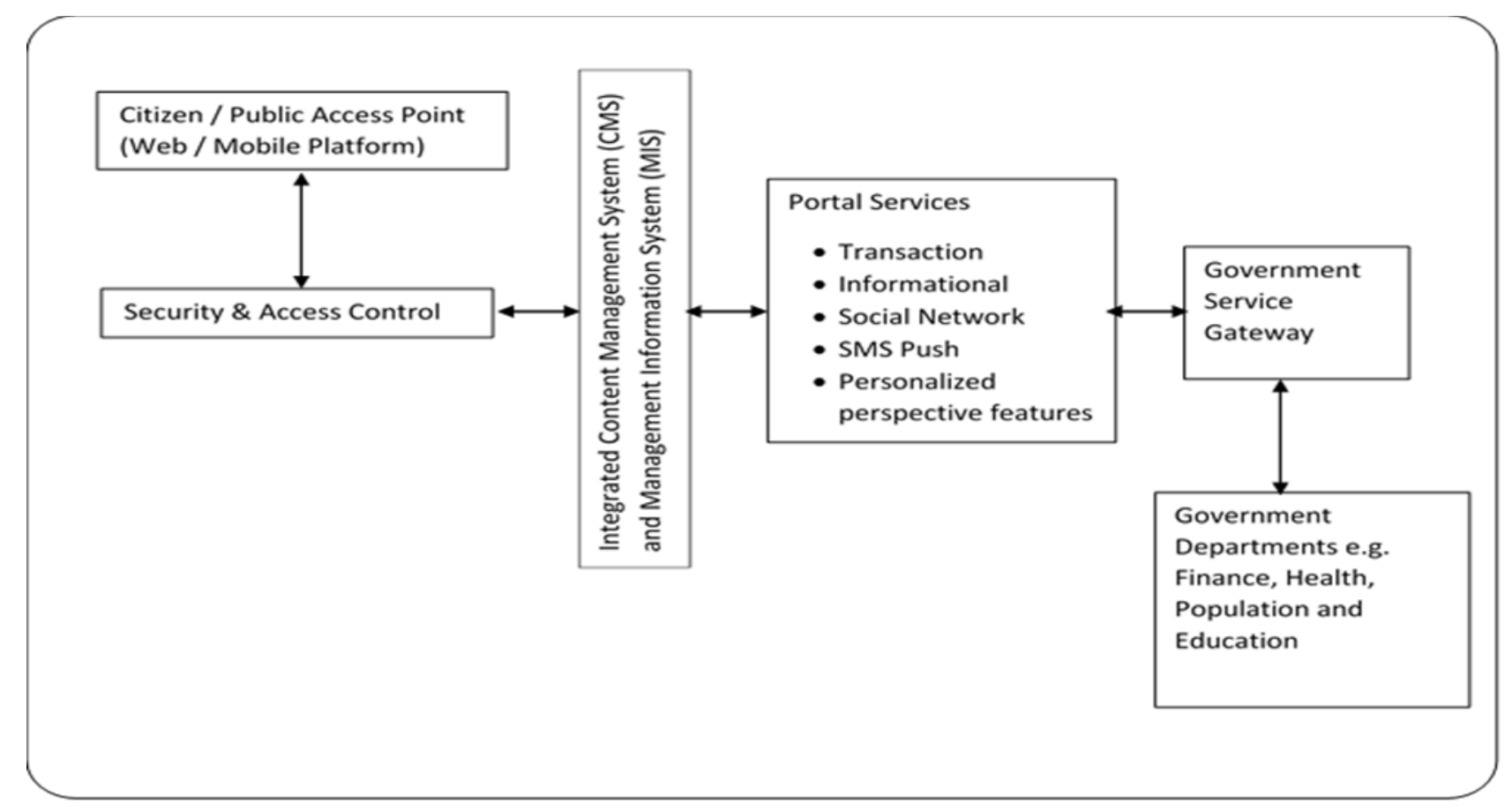

Figure 1: Proposed Framework for OGD Integration to CMS

\section{Citizen/ Public Web and Mobile Platform}

These web browsers and mobile platforms give the citizens an interface that they can use to interact with the government services. The platforms are citizen centered allowing the users to interact free with the OGD datasets.

\section{Security and Access Control}

This is the interface that allows the authentication of users enabling web based single- sign-on and standardized data encryption and digital certificates that can be used for audit trails.

\section{Integrated Content Management Systems and MIS}


The OGD can be integrated with the government content management systems and MIS which are simply designed and have user friendly interfaces. The administrator's interface allows Website content and OGD portal managers and other users to update content without much training in ICT, programming or other technical aspects of system maintenance.

\section{Portal Services}

Transactional Services

This portal allows the public and the citizen to register, apply for birth and death certificates, driving license and issue other departmental services like online payments.

\section{Informational Services}

This portal provides the commonly shared information like public events, news and state departments information.

\section{Social Network Services}

This portal section facilitates the citizen to network and share messages during public discussions like committee boards meeting reports and bulletin. This allows citizens to participate in public interviews, surveys and other activities that will give feedback to the government agencies.

\section{SMS Push Services}

This is used to send messages that is requested by the citizen and enhance public communication. This allows the citizen to be updated on what the government is doing including what services and activities are on progress. This results to transparency and accountability of all the stakeholders.

\section{Personalized Perspective Features}

This enables the OGD portal users to have personalized pages according to the citizen requests. This encourages innovation and creativity of the users of the OGD datasets.

\section{V.CONCLUSION}

The growth in publication of online data through the internet in the last few years has resulted to data explosion. The data produced by the public and the government is reused by citizens, government, researchers, media and other users. Integration of OGD to CMS is expected to result to benefits as discussed in the literature and verified by findings as discussed through the meta data portals analyzed. This study aimed at the existing state of OGD development in Kenya, status of OGD implementation in Kenya, benefits and challenges of OGD integration strategies.

\section{REFERENCES}

[1] Bertot, J. C., Jaeger, P. T., \& Grimes, J. M. (2010). Using ICTs to create a culture of transparency: E-government and social media as openness and anti-corruption tools for societies. Government Information Quarterly, 27(3), 264-271. https://doi.org/10.1016/j.giq.2010.03.001

[2] Burdon, M. (2009). Commercializing public sector information privacy and security concerns. IEEE Technology and Society Magazine, 28(1), 34-40. https://doi.org/10.1109/MTS.2009.931860

[3] Buzzi, M., Ferrucci, L., Gennai, F., \& Petrucci, C. (2016). A proposed evolution for the Italian certified electronic mail system. In eDemocracy \& eGovernment (ICEDEG), 2016 Third International Conference on (pp. 34-41). IEEE. Retrieved from http://ieeexplore.ieee.org/abstract/document/7461693/

[4] Chan, C. M. L. (2013). From Open Data to Open Innovation Strategies: Creating E-Services Using Open Government Data (pp. 18901899). IEEE. https://doi.org/10.1109/HICSS.2013.236

[5] Eden, B. (2009). General Information on Metadata. Library Technology Reports, 38(5), 7-13.

[6] Esperança, C., \& Pereira, A. (2016). Content management system for e-Government portals. In Information Systems and Technologies (CISTI), 2016 11th Iberian Conference on (pp. 1-6). IEEE. Retrieved from http://ieeexplore.ieee.org/abstract/document/7521369/

[7] Government of Kenya. (2013). The Constitution of Kenya: 2010. Chief Registrar of the Judiciary. Retrieved from http://www.wipo.int/edocs/lexdocs/laws/en/ke/ke019en.pdf

[8] Government of Kenya, Second Medium Term Plan 2013-2017. (n.d.).

[9] InternetAccess.pdf. (n.d.). Retrieved from http://www.insidepolitics.org/brookingsreports/InternetAccess.pdf

[10] Janssen, M., Charalabidis, Y., \& Zuiderwijk, A. (2012). Benefits, Adoption Barriers and Myths of Open Data and Open Government. Information Systems Management, 29(4), 258-268. https://doi.org/10.1080/10580530.2012.716740

[11] Janssen, M., \& Estevez, E. (2013). Lean government and platform-based governance-Doing more with less. Government Information Quarterly, 30, S1-S8. https://doi.org/10.1016/j.giq.2012.11.003

[12] Lee, G., \& Kwak, Y. H. (2011). Open Government Implementation Model: A Stage Model for Achieving Increased Public Engagement. In Proceedings of the 12th Annual International Digital Government Research Conference: Digital Government Innovation in Challenging Times (pp. 254-261). New York, NY, USA: ACM. https://doi.org/10.1145/2037556.2037598 
[13] Mutuku, L., \& Mahihu, C. M. (2014). Open Data in Developing Countries. Retrieved from https://opendataresearch.org/sites/default/files/publications/ODDC\%20Report\%20iHub.pdf

[14] Nasr, K. A., Gross, H., \& van Deursen, A. (2010). Adopting and Evaluating Service Oriented Architecture in Industry (pp. 11-20). IEEE. https://doi.org/10.1109/CSMR.2010.13

[15] Nixon Kanali. (2015). Renewing Kenyan driving license online [Blog]. Retrieved February 6, 2017, from http://techtrendske.co.ke/how-torenew-your-kenyan-driving-license-online/

[16] Origlia, C., Cersosimo, D., Bianchi, T., \& Fortunato, A. (2016). Assessing an Open Government Data Initiative. The Case of OpenCoesione (pp. 164-171). IEEE. https://doi.org/10.1109/CeDEM.2016.26

[17] Parycek, P., Höchtl, J., \& Ginner, M. (2014). Open Government Data Implementation Evaluation. Journal of Theoretical and Applied Electronic Commerce Research, 9(2), 13-14. https://doi.org/10.4067/S0718-18762014000200007

[18] Public Governance and Territorial Development directorate public governance committee. (n.d.). Retrieved from http://www.oecd.org/officialdocuments/publicdisplaydocumentpdf/?cote=GOV/PGC/EGOV(2012)7/REV1\&docLanguage=En

[19] Sebastian Meumanier, Open Data Quality Assesment and Evaluation of (Meta-) Data Quality in the Open Data Landscape. (n.d.).

[20] Weseni, T. A., Watson, R. T., \& Anteneh, S. (2015). A review of soft factors for adapting public-private partnerships to deliver public information services in Ethiopia: A conceptual framework. In AFRICON, 2015 (pp. 1-6). IEEE. Retrieved from http://ieeexplore.ieee.org/abstract/document/7332016/

[21] Yunliang, J., Xiongtao, Z., Qing, S., Jing, F., \& Ning, Z. (2010). Design of E-Government Information Management Platform Based on SOA Framework (pp. 165-169). IEEE. https://doi.org/10.1109/ICNDC.2010.42 